\title{
Changes in the carbon monoxide diffusing capacity of the lung in ulcerative colitis
}

\author{
M. Marvisi*, P.D. Borrello*, M. Brianti*, G. Fornarsari**, G. Marani*, A. Guariglia*
}

Changes in the carbon monoxide diffusing capacity of the lung in ulcerative colitis. $M$. Marvisi, P. del Borrello, M. Brianti, G. Fornarsari, G. Marani, A. Guariglia. (C)ERS Journals Ltd 2000.

ABSTRACT: The aim of this study was to investigate lung function in patients with ulcerative colitis and to assess the incidence of latent pulmonary involvement in subjects with active and inactive disease.

After full colonscopic assessment with multiple mucosal biopsy, the clinical disease activity of each patient was quantified, using the simple index of Harvey and Bradshaw. The patients were divided into 2 equal groups: subjects with active disease (group 1; $n=16$ ); and those with inactive disease (group 2; $n=16$ ). Global spirometry was then performed.

A latent pulmonary involvement was found in 17 of 32 patients $(53 \%)$, the incidence was higher in the group 1 patients $(81 \%)$. The majority of patients presented a reduction in the carbon monoxide diffusing capacity of the lungs $(D \mathrm{~L}, \mathrm{CO})$. The mean $D L, C O$ value was $73.87 \pm 14.87$ in group 1 and $87.31 \pm 11.23$ in group 2 . The $D L, C O$ and $K$ CO reduction correlated significantly with intestinal histopathological grading in the group of patients with active disease $(r=0.87, p<0.001 ; r=0.603, p=0.015)$.

To conclude, a high incidence of pulmonary function abnormalities were identified, despite the lack of radiological alterations (High Resolution Computed Tomography) and pulmonary symptoms, in ulcerative colitis patients. These alterations were more common in patients with active disease. The strong correlation between $D \mathrm{~L}, \mathrm{CO}$ values and histopathological grading suggests that this test may reflect bowel disease activity. Eur Respir J 2000; 16: 965-968.
*Dept of Internal Medicine and **Division of General Surgery, FiorenzuolaCortemaggiore Hospital, Piacenze, Italy.

Correspondence: M. Marvisi

Cortemaggiore Hospital

Cortemaggiore

via Liberta' 6-Piacenza

Italy, 29016

Fax: 390523832860

Keywords: Carbon monoxide diffusing capacity

inflammatory bowel disease

latent pulmonary involvement

lung disease

pulmonary function

ulcerative colitis

Received: April 32000

Accepted after revision August 32000

Presented, in part, at the European Respiratory Society annual congress, Stockholm (Sweden), 1996.
Ulcerative colitis (UC) is a systemic illness with a number of extraintestinal manifestations affecting various organs. The most frequent lesions are cutaneous (pyoderma gangrenosum, erythema nodosum), ocular (anterior uveitis, episcleritis), hepatic (pericholangitis, fatty liver), articular (peripheral and axial arthropathy). In contrast, pulmonary involvement in UC is thought to be rare. The first author to describe a possible association of UC and respiratory disease was TURNER-WARWICK [1] in 1968, but it was not until KRAFT et al. [2] published their work in 1976 that respiratory involvement was included in the list of established complications of inflammatory bowel disease (IBD).

The majority of patients, reported in the English language literature, have airways disease with chronic bronchitis and bronchiectasis, but other manifestations were described including: interstitial pneumonitis, panbronchiolitis, bronchiolitis obliterans organizing pneumonia (BOOP), inflammatory tracheal stenosis, serositis, pulmonary vasculitis, apical fibrosis and conditions resembling Wegener's granulomatosis [3]. Some authors described alterations of pulmonary function in asymptomatic patients, most commonly reduced lung diffusion capacity and small airways function and bronchial hyperreactivity [4-6].

The aim of the present study was to investigate lung function in patients with UC and to assess the incidence of latent pulmonary involvement in subjects with active and inactive disease. To the best of the authors knowledge this information is not available in the literature.

\section{Materials and methods}

Thirty two patients with UC (18 males and 14 females), aged $45.75 \pm 12.05$ (mean \pm standard deviation (SD)) undergoing full colonscopic assessment were studied (table 1). The diagnosis was based on clinical and morphological data. Multiple mucosal biopsy specimens were taken from the macroscopically affected regions to establish the severity of the disease activity. The duration of illness was $167.44 \pm 150.05$ months. All patients were nonsmokers, had an insignificant occupational history, no previous viral and bacterial lung infections and had a Body Mass Index $<30 \mathrm{~kg} \cdot \mathrm{m}^{2}$.

There was no clinical evidence of connective tissue diseases and all subjects had a negative antinuclear antibody (ANA) test. Exclusion criteria were the presence of pulmonary, cardiac and renal diseases performing a highresolution computed tomography (HRCT), transthoracal doppler echocardiography (Esaote Sim 7000 Challenger, Florence, Italy) and a valuation of creatinine clearance. All patients were receiving 5-aminosalicylate (5-ASA) treatment only, and four subjects were receiving concomitant antihypertensive drugs (table 2). 
Table 1. - Patients characteristics

\begin{tabular}{lccc}
\hline & Group 1 & Group 2 & p-value \\
\hline SEX M/F & $9 / 7$ & $9 / 7$ & NS \\
Age yr & $44.81 \pm 13.94$ & $46.69 \pm 10.16$ & NS \\
$\begin{array}{l}\text { Disease } \\
\text { dur.wks }\end{array}$ & $163.31 \pm 185.02$ & $171.58 \pm 115.08$ & 0.879 \\
TLC \% & $97.69 \pm 12.83$ & $98.80 \pm 12.94$ & 0.946 \\
FEV1 \% & $98.19 \pm 11.59$ & $99.31 \pm 13.70$ & 0.799 \\
FVC \% & $97.87 \pm 10.68$ & $97.37 \pm 12.47$ & 0.904 \\
F50-75\% & $72.00 \pm 22.60$ & $88.31 \pm 11.71$ & 0.073 \\
DL,CO \% & $73.87 \pm 14.27$ & $87.31 \pm 11.23$ & 0.003 \\
KCO \% & $77.50 \pm 15.05$ & $87.62 \pm 13.25$ & 0.050 \\
BMI & $21.5 \pm 2.4$ & $22.1 \pm 2.7$ & NS \\
ESR mm & $66.31 \pm 16.86$ & $20.06 \pm 9.77$ & $<0.001$ \\
CRP & $4.38 \pm 2.00$ & $1.89 \pm 0.88$ & $<0.001$ \\
5-ASA & $2956.25 \pm 848.50$ & $2968.75 \pm 732.77$ & 0.965 \\
mg.dL $^{-1}$ & & & \\
\hline
\end{tabular}

Data are presented as mean \pm SD. TLC: total lung capacity; FEV1: forced expiratory volume in one second; FVC: forced vital capacity; F50-75\%: flows at $50-75 \%$ of FVC; DL,CO: carbon monoxide diffusing capacity of the lung; KCO: carbon monoxide transfer coefficient; BMI: body mass index; ESR: erythrocite sedimentation rate; CRP: C-reactive protein; Disease dur.wks: disease duration in weeks; 5-ASA: 5 aminosalicylate. ns: not significant.

On the day of endoscopy, clinical disease activity was quantified using the simple index of Harvey and Bradshaw (HBI) [7]. All patients had venesection for routine biochemistry tests and for the estimation of C-reactive protein $(\mathrm{CRP}$, immunological turbidity test, normal value $=$ $0-0.5 \mathrm{mg} \cdot \mathrm{dL}^{-1}$ ) erythrocyte sedimentation rate (ESR) and antineutrophil cytoplasmic antibodies (ANCA enzymelinked immunosorbent assays).

Each biopsy specimen was assessed blindly by one histopathologist with a special interest in gastrointestinal diseases, who was unaware of the disease activity index of each case. In each specimen, scores were assigned to the severity of surface enterocyte damage, cryptitis and acute and chronic inflammation in the lamina propria (table 3). A global spirometry was performed in all patients using a
Table 3. - Histological assessment of mucosal biopsy specimens

\begin{tabular}{lc}
\hline Specimen & Grade \\
\hline Enterocytes & \\
Normal & 0 \\
Loss of single cell & 1 \\
Loss of groups of cells & 2 \\
Frank ulceration & 3 \\
Crypts & \\
Normal & 0 \\
Single inflammatory cells & 1 \\
Cryptitis & 2 \\
Crypt abscesses & 3 \\
Lamina propria mononuclear cells & \\
Normal & 0 \\
Mild increase & 1 \\
Moderate increase & 2 \\
Marked increase & 3 \\
Neutrophils & \\
Normal & 0 \\
Mild increase & 1 \\
Moderate increase & 2 \\
Marked incease & 3 \\
\hline
\end{tabular}

Conversion of histological scores to grades was carried out according to the following (the data is presented as Grade (Total score). 0 (0-1); 1 (2-4); 2 (5-8); 3 (8-10); 4 (10-12).

dry wedge spirometer (Vitalograph, PK Morgan Ltd, UK), lung volumes were determined by a closed circuit helium dilution technique, DL,CO was measured by the single-breath method and corrected by alveolar volume $(V \mathrm{~A})$, using a Morgan Transfer machine model $\mathrm{C}(\mathrm{PK}$ Morgan Ltd, UK). Haemoglobin was measured because of its influence on the $D \mathrm{~L}, \mathrm{CO} / V \mathrm{~A}(K \mathrm{CO})$ values. The patients were divided into two equal groups: group 1 $(\mathrm{n}=16)$, subjects with active disease $(\mathrm{HBI}$ index $>4)$ and group $2(\mathrm{n}=16)$, subjects with inactive disease (HBI index $<4)$. All patients gave written informed consent after the purpose of the study had been explained.

Table 2. - Characteristics of patients with active disease (group 1)

\begin{tabular}{|c|c|c|c|c|c|c|c|}
\hline Age yr & Grading & Systemic involvement & Drugs* & $D \mathrm{~L}, \mathrm{CO} \%$ & $\mathrm{~F} 50-75 \%$ & ESR & CRP \\
\hline 48 & 9 & none & 5-ASA & 71 & 30 & 61 & 2 \\
\hline 21 & 8 & erythema nodosum & 5-ASA & 73 & 89 & 70 & 2.5 \\
\hline 55 & 12 & none & 5-ASA+Ena & 65 & 40 & 55 & 4 \\
\hline 50 & 11 & none & 5-ASA & 63 & 100 & 81 & 3.5 \\
\hline 32 & 12 & Fatty liver & 5-ASA & 60 & 83 & 69 & 2.5 \\
\hline 41 & 11 & none & 5-ASA & 64 & 77 & 80 & 5 \\
\hline 20 & 9 & none & 5-ASA & 78 & 54 & 51 & 3 \\
\hline 60 & 11 & none & 5-ASA+Dox & 65 & 64 & 48 & 3.5 \\
\hline 59 & 11 & Fatty liver & 5-ASA & 62 & 78 & 90 & 4 \\
\hline 58 & 8 & Pyoderma & 5-ASA+Quin & 76 & 95 & 88 & 5 \\
\hline 27 & 10 & none & 5-ASA & 67 & 58 & 49 & 3.5 \\
\hline 41 & 11 & none & 5-ASA & 67 & 48 & 59 & 4.5 \\
\hline 39 & 9 & anterior uveitis & 5-ASA & 70 & 89 & 91 & 7.5 \\
\hline 47 & 5 & none & 5-ASA & 99 & 99 & 79 & 8.5 \\
\hline 58 & 6 & none & 5-ASA & 105 & 55 & 50 & 8 \\
\hline 61 & 5 & none & 5-ASA+Ram & 98 & 97 & 46 & 3 \\
\hline
\end{tabular}

*: 5-ASA used at 1,600-4,000 mg.day ${ }^{-1}$. Ena: enalapril; Dox: doxazosin; Quin: quinapril; Ram: Ramipril; DL,CO: carbon monoxide diffusing capacity of the lung; F50-75: flows at 50-75\% of forced vital capacity; ESR: erythrocyte sedimentation rate; CRP: C-reactive protein. 
Table 4. - Correlations with $D \mathrm{~L}, \mathrm{CO}$ in patients with active ulcerative colitis (group 1)

\begin{tabular}{lcr}
\hline & R-value & P-value \\
\hline DL,CO-Grading & 0.875 & $<0.001$ \\
$D$ L,CO-ESR & 0.296 & 0.263 \\
DL,CO-CRP & 0.235 & 0.373 \\
DL,CO-Age & 0.033 & 0.900 \\
$D$ L,CO-5-ASA & 0.304 & 0.244 \\
\hline
\end{tabular}

$D$ L,CO: carbon monoxide diffusing capacity of the lung; ESR: erythrocyte sedimentation rate; CRP: C-reactive protein; 5ASA: 5-aminosalicylate dosage.

\section{Statistical method}

All data are expressed as mean \pm SD. Statistical comparisons were made using a paired t-test for total lung capacity (TLC), forced expiratory volume in one second (FEV1) and forced vital capacity (FVC). The other variables were evaluated using the nonparametric Mann-Whitney U-test.

The correlations between spirometric data and histological grading were made using the Spearman rank sum test. A probability value of $<0.05$ was considered to be statistically significant.

\section{Results}

A latent pulmonary involvement was found in 17 of 32 patients with UC (53\%). The incidence was higher in patients with active disease (group 1), 13 of $16(81 \%)$. The majority of patients presented a mild reduction in both $D \mathrm{~L}, \mathrm{CO}$ and F25-75 (56\%), $25 \%$ of $D \mathrm{~L}, \mathrm{CO}$ only. The mean $D$ L,CO value was $73.87 \pm 14.87$ in group 1 and $87.31 \pm 11.23$ in group 2 ( $\mathrm{p}=0.003)$. Similarly, the mean $K \mathrm{CO}$ value was greater in patients with inactive rather than active disease (87.62 \pm 13.25 versus $77.50 \pm 15.05 ; \mathrm{p}=0.050)$. A mild, but not statistically significant difference in the mean F50-75 value was found between patients with active and those with inactive UC $(72.00 \pm 22.60$ versus $88.31 \pm 11.71$; $\mathrm{p}=$ 0.073 ). Nonsignificant differences in the mean FVC, FEV1 value, TLC and FEV1\FVC values were found between patients with active and inactive UC (table 1). The histopathological grading showed a strong positive correlation with the clinical disease activity $(\mathrm{r}=0.80 ; \mathrm{p}<0.001)$.

$D$ L,CO reduction correlated significantly with intestinal histopathological grading in group $1(\mathrm{r}=-0.875 ; \mathrm{p}<0.001)$ (table 4$)$ but not in group $2(\mathrm{r}=0.014 ; \mathrm{p}=0.952)$. KCO also correlated significantly with histopathological grading in group $1(\mathrm{r}=0.603 ; \mathrm{p}=0.015)$. No significant correllation was seen between grading and F50-75 ( $\mathrm{r}=-0.320$; $\mathrm{p}=$ 0.221 , in group $1 ; \mathrm{r}=0.221 ; \mathrm{p}=0.404$ in group 2 ), or the 5 -ASA dosage and F50-75 value $(\mathrm{r}=0.232, \mathrm{p}=0.379)$. Similarly, no significant correlations were found between $D$ L,CO and 5-ASA dosage ( $\mathrm{r}=0.304, \mathrm{p}=0.244), D \mathrm{~L}, \mathrm{CO}$ and ERS. DL,CO and CRP, DL,CO and age. ERS and histopathological grading $(\mathrm{r}=0.115 ; \mathrm{p}=0.664)$, or $\mathrm{CRP}$ and grading $(\mathrm{r}=-0.160 ; \mathrm{p}=0.373)$ (table 4$)$. In the present study, a positive ANCA test with a perinuclear staining pattern (p-ANCA), was found in only one group 2 patient (titre 1:160).

\section{Discussion}

In the series of cases in this study a high incidence of pulmonary function abnormalities were identified, despite the lack of radiological alterations and pulmonary symptoms. The data is in accordance with the recently reported study by Kuzela et al. [4] who found a reduction in the $D$ L,CO in $56.7 \%$ patients with UC and in $57.7 \%$ of those with Crohn's disease. Godet et al. [8] studied 55 patients with UC and found alterations in $30(55 \%)$ subjects, these alterations could not be predicted by current or past smoking status, family history of respiratory disease, occupational history or current medication use. TZANAKIS et al. [5] who studied a larger group of patients, reported a lower incidence of $D \mathrm{~L}, \mathrm{CO}$ reduction $(17.6 \%)$ but a similar correlation between $D \mathrm{~L}, \mathrm{CO}$ reduction and active phase of the disease. Munck et al. [6] arrived at the same conclusions when studying a group of paediatric patients with Crohn's disease.

To the best of the authors knowledge, the present study is the only one that used HRTC to exclude macroscopic interstitial fibrosis or alveolar and interstitial inflammation. On the other hand, a mild but not statistically significant reduction of F50-75 in patients with active UC was found. TZANAKIS et al. [9] who performed maximal expiratory flow volume curves while breathing room air and a mixture of $80 \%$ helium and $20 \%$ of oxygen, demonstrated that the latter method could show a latent small peripheral airways involvement in patients with IBD. Evidence for the presence of subclinical airways involvement can also be obtained from the report by Louis et al. [10] they found an increased nonspecific bronchial hyperresponsiveness to methacoline, independent from the presence of atopy, in patients with IBD and normal baseline spirometry [10].

In addition, the present study, is the first, to the authors knowledge that shows a strong correlation between $D \mathrm{~L}, \mathrm{CO}$ (and $K \mathrm{CO}$ ) values and histopathological grading. However, $D$ L,CO abnormalities were not correlated with serological and biochemical indices of disease activity like ERS and CRP. Other authors found no significant correlation between microscopic score and ERS/CRP, these tests, considered separately, are nonspecific, yielding values that depend on certain serum proteins (most notably fibrinogen) [11, 12]. Recently, MAHMUD et al. [13] demonstrated a similar correlation between histological grading and microalbuminuria (measured using the immunoturbiditimetric method). They suggested that this simple, inexpensive, and noninvasive index that accurately reflects histological disease activity is clearly of importance in monitoring the progress of a patient's illness. The same concept may also be valid for $D \mathrm{~L}, \mathrm{CO}$.

At this juncture, the following question still needs answering. Is the lung a target organ in ulcerative colitis and in inflammatory bowel disease? The reply seems to be positive. The pathogenesis of UC causing lung abnormalities is unkown, but both morphologic and developmental similarities exist between colonic and bronchial epithelium; both are derived from primitive gut and have columnar epithelia with goblet cells and submucosal mucus glands [14]. On the other hand, the activated inflammatory cells in bowel tissues are capable of producing a number of circulating cytokines such as interleukin 1, interleukin 2, interleukin 6 and tumour necrosis factor- $\alpha$ [15]. These and other mediators can 
regulate the endothelial cell adhesion molecules, alter white cell migration, increase the production of damaging reactive oxygen metabolites and induce damage of lung parenchyma. The findings of studies using bronchoalveolar lavage fluid lend additional support to this hypothesis, in fact high proportions of alveolar lymphocytes have been reported in patients with IBD who are free of pulmonary symptoms and have normal chest radiographs. In this series of cases a low incidence of positive p-ANCA test was found and the authors suggest that the pathogenetic contribution of these antibodies, for instance in terms of neutrophils enzymatic release and tissue damage, needs to be clarified.

The study indicates that subclinical alveolitis and/or interstitial lung disease may be present in patients with active ulcerative colitis, since it is known that a reduction in the diffusing capacity of the lungs is an early manifestation of interstitial disease [16, 17]. Furthermore, the carbon monoxide diffusing capacity of the lung may provide an additional noninvasive indicator of colonic inflammation in patients with ulcerative colitis.

\section{References}

1. Turner-Warwick M. Fibrosing alveolitis and chronic liver disease. OJM 1968; 37: 133-149.

2. Kraft SC, Earle RH, Roesler M, Esterly JR. Unexplained bronchopulmonary disease with inflammatory bowel disease. Arch Intern Med 1976; 136: 454-459.

3. Camus P, Piard F, Aschoft T, Gal AA, Colby TV. The lung in inflammatory bowel disease. Medicine 1993; 72: 151153.

4. Kuzela L, Vavrecka A, Prikazska M, et al. Pulmonary complications in inflammatory bowel disease. Hepatogastroenterology. 1999; 46: 1714-1719.

5. Tzanakis N, Bouros D, Samiou M, et al. Lung function in patients with inflammatory bowel disease. Resp Med 1998; 98: 516-522.

6. Munck A, Murciano D, Pariente R, Cezard J, Navarro J.
Latent pulmonary abnormalities in children with Crohn's disease. Eur Respir J 1995; 8: 377-380.

7. Harvey RF, Bradshaw JW. A simple index of Crohn's disease activity. Lancet 1980; i: 514.

8. Godet PG, Cowie R, Woodman RC, Sutherland LR. Pulmonary function abnormalities in patients with ulcerative colitis. Am J Gastroenterol 1997; 92: 11541156.

9. Tzanakis N, Samiou M, Bouros D, Mouzas J, Kouroumalis E, Siafakas MN. Small airway function in patients with inflammatory bowel disease. Am J Respir Crit Care Med 1998; 157: 382-386.

10. Louis E, Louis R, Drion V, Bonnet V, Lamproye A, Radermecker M. Increased frequency of bronchial hyperresponsiveness in patients with inflammatory bowel disease. Allergy 1995; 50: 728-733.

11. Cellier C, Sahamoud T, Froguel E, et al. Correlation between clinical activity, endoscopic severity and biological parameters in colonic or ileocolonic Crohn's disease. A prospective multicentre study of 121 cases. Gut 1994; 35: 231-235.

12. Gomes P, Boulay CD, Smith CL, Holdstock G. Relationship between disease activity indices and colonscopy findings in patients with colonic inflammatory bowel disease. Gut 1986; 27: 92-95.

13. Mahamud N, McDonald GSA, Keller D, Weir DG. Microalbuminuria correlates with intestinal histopathological grading in patients with inflammatory bowel disease. Gut 1996; 38: 99-103.

14. Cohen M, Sahn SA. Bronchiectasis in systemic disease. Chest 1999; 116: 1063-1074.

15. Mitsuyama K, Sasaki E, Toyanaga A, et al. Colonic mucosal interleukin 6 in inflammatory bowel disease. Digestion 1991; 50: 104-111.

16. Andus T, Gross V, Casar D, et al. Activation of monocytes during inflammatory bowel disease. Pathobiology 1991; 59: 166-170.

17. Wallaert B, Dugas M, Dansin $\mathrm{T}$, et al. Subclinical alveolitis in immunological systemic disorders. Transition between health and disease. Eur Respir J 1990; 3: 12061216. 